\title{
Uso de peças cadavéricas e modelos sintéticos no ensino da anatomia nos cursos de enfermagem
}

Use of cadaveric pieces and synthetic models in the anatomy teaching in undergraduate nursing courses

Uso de piezas cadavéricas y modelos sintéticos en la enseñanza de la anatomía en los cursos de enfermería

\section{Nathan Mesquita Penha ${ }^{\mathrm{I1}}$, Laura Menezes Silveira ${ }^{\mathrm{II}}$, Fernanda dos Santos Nogueira de Góes ${ }^{\mathrm{III}}$, Angelita Maria Stabile ${ }^{\mathrm{IV}}$}

\begin{abstract}
Resumo: Objetivo: identificar potencialidades e dificuldades do uso de peças cadavéricas e sintéticas no ensino de anatomia nos cursos de Enfermagem. Método: estudo quantitativo, transversal e descritivo. Um questionário contendo 23 perguntas foi enviado aos estudantes do curso de Enfermagem que finalizaram a disciplina de anatomia nos anos de 2015 e 2016. Os dados foram analisados com auxílio de software estatístico denotados por frequência absoluta e relativa. Resultados: participaram do estudo 132 estudantes, destes 80,3\% concordaram que as peças cadavéricas ajudam na construção do conhecimento; 35,6\% não apresentam dificuldades em relacionar peças sintéticas com as cadavéricas; $38,6 \%$ discordam que o uso somente de peças cadavéricas é suficiente para a aprendizagem e 55,3\% consideram que a utilização concomitante das peças auxilia o entendimento. Conclusão: o uso de cadáveres no ensino da Anatomia apresenta mais potencialidades quando comparado ao uso de peças sintéticas. A utilização associada desses recursos facilita o processo de ensino-aprendizagem.
\end{abstract}

Descritores: Anatomia; Enfermagem; Cadáver; Educação superior; Educação em enfermagem

Abstract: Objective: to identify potentialities and difficulties of the use of cadaveric and synthetic pieces in the anatomy teaching in Nursing courses. Method: quantitative, cross-sectional and descriptive study. A questionnaire containing 23 questions was sent to the students of the Nursing course who finished the anatomy subject in 2015 and 2016. Data were analyzed with the help of statistical software denoted by absolute and relative frequencies. Results: the study included 132 students, $80.3 \%$ of them agreed that cadaveric pieces help the knowledge construction; $35.6 \%$ do not have difficulties in relating synthetic parts with cadaveric ones; $38.6 \%$ disagree that the use of cadaveric pieces alone is enough for learning; and $55.3 \%$ consider that the concomitant use

\footnotetext{
${ }^{I}$ Enfermeiro, Graduação em Enfermagem, Escola de Enfermagem de Ribeirão Preto - Universidade de São Paulo (EERP/USP), Ribeirão Preto, São Paulo, Brasil. E-mail: Nathan.penha@outlook.com, https://orcid.org/0000-0001-5159-7319

II Enfermeira, Mestre em Ciências, Doutoranda, Escola de Enfermagem de Ribeirão Preto - Universidade de São Paulo (EERP/USP), Ribeirão Preto, São Paulo, Brasil. E-mail: lauramsilveira@usp.com.br, https://orcid.org/0000-0002-2397-2553

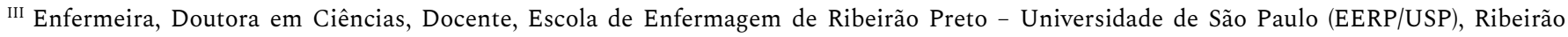
Preto, São Paulo, Brasil. E-mail: fersngoes@eerp.usp.br, https://orcid.org/0000-0001-6658-916X

IV Enfermeira, Doutora em Ciências, Docente, Escola de Enfermagem de Ribeirão Preto - Universidade de São Paulo (EERP/USP), Ribeirão Preto, São Paulo, Brasil. E-mail: angelita@eerp.usp.br, https://orcid.org/0000-0002-3371-7038
} 
of pieces helps the understanding. Conclusion: the use of cadavers in the anatomy teaching shows more potentialities when compared to the use of synthetic pieces. The associated use of these resources facilitates the teaching-learning process.

Descriptors: Anatomy; Nursing; Cadaver; Higher education; Nursing education

Resumen: Objetivo: identificar las potencialidades y dificultades del uso de piezas cadavéricas y sintéticas en la enseñanza de la anatomía en los cursos de enfermería. Método: estudio cuantitativo, transversal y descriptivo. Se envió un cuestionario con 23 preguntas a los estudiantes del curso de Enfermería que terminaron la asignatura de anatomía en 2015 y 2016. Los datos se analizaron con la ayuda de un programa estadístico denotados por frecuencias absolutas y relativas. Resultados: el estudio abarcó 132 estudiantes, el 80,3\% de ellos acordaron que las piezas cadavéricas ayudan a la construcción del conocimiento; el 35,6\% no presentaron dificultades para relacionar las partes sintéticas con las cadavéricas; el 38,6\% discreparon con el uso solamente de las piezas cadavéricas es suficiente para el aprendizaje; y el 55,3\% consideran que el uso concomitante de las piezas ayuda a la comprensión. Conclusión: el uso de cadáveres en la enseñanza de la Anatomía presenta mayores potencialidades en comparación con el uso de piezas sintéticas. El uso asociado de estos recursos facilita el proceso de enseñanza-aprendizaje.

Descriptores: Anatomía; Enfermería; Cadáver; Educación superior; Educación en enfermería

\section{Introdução}

A anatomia é a ciência que estuda a estrutura do corpo humano. O termo anatomia vem da palavra grega Anatome que significa “cortar em partes" (ana = cortar; tome = em partes). Os primeiros registros sobre dissecação de cadáveres humanos são do século XIX a.C. no Egito antigo. ${ }^{1}$

O ensino da anatomia ocorre a partir da observação direta de peças e cortes de estruturas anatômicas com o objetivo de relacioná-las com a sua função e com as modulações de estrutura em resposta a fatores temporais, genéticos e ambientais. ${ }^{2}$ A anatomia é uma disciplina básica para todos os estudantes ingressantes na área da saúde. Especialmente, nos cursos de graduação em enfermagem, ela é a base para o entendimento de outras disciplinas fundamentais, como a fisiologia, patologia e outras associadas ao cuidado do ser humano..$^{3-4}$ Além disso, o ensino da anatomia pode contribuir para a formação do comportamento ético do enfermeiro. ${ }^{5}$

Para que o conhecimento da anatomia se concretize, são aplicados diversos métodos de ensino. O método mais tradicional na área da saúde é estruturado em dois momentos distintos: 
o primeiro, na sala de aula, onde são apresentados conceitos e definições dos diferentes sistemas e órgãos do corpo humano, suas características inter-relações; no segundo momento, são realizadas as aulas práticas em laboratórios, utilizando-se peças anatômicas naturais (cadavéricas) e/ou sintéticas que sejam capazes de auxiliar no processo de ensinoaprendizagem. ${ }^{6}$

Além do uso de peças cadavéricas, a utilização de modelos anatômicos artificiais é uma prática comum nos cursos de graduação da área da saúde. ${ }^{6}$ Esses recursos inovadores e alternativos visam suprir as desvantagens que são apresentadas com o uso de cadáveres, como quantidade insuficiente, dificuldade de aquisição, alto custo para a sua manutenção, dificuldades de armazenamento adequado, degradação causada pelo manuseio constante e o emprego de produtos químicos e tóxicos para sua conservação. ${ }^{7}$ Adicionalmente, outras ferramentas como e-learning, áudios, vídeos, jogos e realidade tridimensional têm sido empregadas para complementar o ensino da Anatomia. ${ }^{6-9}$

Apesar de, o uso de cadáveres ser reconhecidamente necessário para o ensino da anatomia, ${ }^{6-9}$ o uso de peças sintéticas e outras tecnologias pode colaborar com o aprendizado. Ademais, muitos estudantes se mostram apreensivos antes de ver o cadáver pela primeira vez, e podem apresentar mal-estar, fraqueza e náusea ao entrar em contato com ele. ${ }^{10}$ Estudos mostram que é relevante atentar-se as preferências dos estudantes, ${ }^{6}$ as facilidades de cada recurso didático e as contribuições do uso de cadáveres e de tecnologias no ensino da anatomia. ${ }^{9}$ Contudo, essas evidências são majoritariamente provenientes de pesquisas realizadas com estudantes da área médica ${ }^{6,8-9}$ e, em relação à enfermagem, há escassez de estudos que abordam tais características. ${ }^{3,5}$

O ensino da anatomia para a enfermagem compõe o “eixo básico” para a compreensão das estruturas anatômicas (forma e localização) o que permite a comparação com as estruturas do indivíduo vivo; promove melhor entendimento da fisiopatologia, da avaliação clínica, e da 
realização de procedimentos de enfermagem. Assim, visando o acesso aos aspectos que possam vir a contribuir para uma melhor aplicação de novos recursos no ensino, tendo em vista não só o ensino-aprendizagem mais efetivo como também a formação de um profissional qualificado, este estudo questionou quais as potencialidades e dificuldades no uso de peças cadavéricas e sintéticas no ensino da anatomia nos cursos de enfermagem? O objetivo foi identificar as potencialidades e dificuldades do uso de peças cadavéricas e peças sintéticas no ensino da anatomia nos cursos de enfermagem.

\section{Método}

Trata-se de um estudo quantitativo, transversal e descritivo. A população foi composta por estudantes de graduação dos cursos de Enfermagem: Bacharelado (80 estudantes/ano) e Bacharelado e Licenciatura (50 estudantes/ano) de uma instituição de ensino superior do interior do Estado de São Paulo, que finalizaram a disciplina de Anatomia nos anos de 2015 e 2016, independente de terem ou não sido aprovados. Foram enviados 260 convites.

$\mathrm{Na}$ referida instituição, a anatomia é uma disciplina que compõe a grade do primeiro ano dos cursos. O ensino de anatomia é caracterizado pelo método de ensino de anatomia em sistemas, os quais são apresentados aos estudantes em aulas teóricas e práticas. Neste modelo didático, a primeira aula prática de cada sistema é realizada com peças sintéticas. De forma dinâmica, os estudantes são preparados para identificar e conhecer a topografia corporal interrelacionando-a com as funções fisiológicas. A segunda aula prática, por sua vez, é realizada com peças cadavéricas conservadas por meio do processo de formalização.

Inicialmente, os estudantes foram convidados via correio eletrônico. No convite, haviam explicações acerca da finalidade do estudo e a indagação sobre qual seria a melhor data e horário para aplicação do questionário. 
Para a coleta de dados, foi elaborado um questionário estruturado (do tipo Survey) composto por questões fechadas, previamente estabelecidas, capazes de identificar a avaliação dos estudantes quanto às potencialidades e dificuldades do uso de peças cadavéricas e modelos sintéticos para o ensino da anatomia na área de enfermagem. Esse questionário foi elaborado com base na literatura científica, ${ }^{11-12}$ estruturado em três partes, contendo 23 perguntas e respostas por meio de uma escala de Likert de cinco alternativas. A primeira parte do questionário avaliou o uso de peças cadavéricas, a segunda parte o uso de peças sintéticas e a terceira parte o uso concomitante dessas no ensino da anatomia. As questões versavam sobre a facilidade de manuseio e a representação fidedigna das estruturas, facilitando o alcance dos objetivos de aprendizagem.

O instrumento foi avaliado por um comitê formado por três especialistas com experiência no ensino de enfermagem, os quais avaliaram o conteúdo do instrumento, observando em que extensão o assunto de interesse era contemplado pelos itens, analisando se esses estavam adequados ao universo do conteúdo que definia as variáveis de interesse desse estudo. Os questionários, juntamente com o Termo de Consentimento Livre e Esclarecido (TCLE) foram entregues para os interessados em participar da pesquisa em data e horário marcados.

As respostas obtidas foram digitadas duplamente no programa Microsoft Office Excel 2010 e exportadas para o software estatístico Statistical Package for the Social Sciences (SPSS), sendo calculadas a frequência absoluta e frequência relativa, das variáveis investigadas no estudo. Os resultados foram apresentados em tabelas. Este estudo foi elaborado de acordo com as diretrizes contidas na Resolução CNS 466/2012 para o desenvolvimento de pesquisa com seres humanos e autorizado pelo Comitê de Ética em Pesquisa com Seres Humanos, aprovado conforme número CAAE: 52683215.7.0000.5393 no dia sete de março de 2016. 
Uso de peças cadavéricas e modelos sintéticos no ensino da anatomia nos... | 6

\section{Resultados}

Participaram desse estudo 132 estudantes de graduação em Enfermagem. Desses, 57 $(43,2 \%)$ eram graduandos do curso de Bacharelado e $75(56,8 \%)$ do curso de Bacharelado e Licenciatura em Enfermagem. Entre os graduandos, $115(87,1 \%)$ eram mulheres e $17(12,9 \%)$ homens.

A Tabela 1 mostra as respostas relativas às características da primeira parte do questionário que avaliou as potencialidades e dificuldades do uso de peças cadavéricas no ensino da anatomia no curso de graduação em Enfermagem.

Tabela 1 - Avaliação dos estudantes de enfermagem quanto ao uso de peças cadavéricas no ensino da Anatomia, Ribeirão Preto/SP, Brasil, 2016-2017.

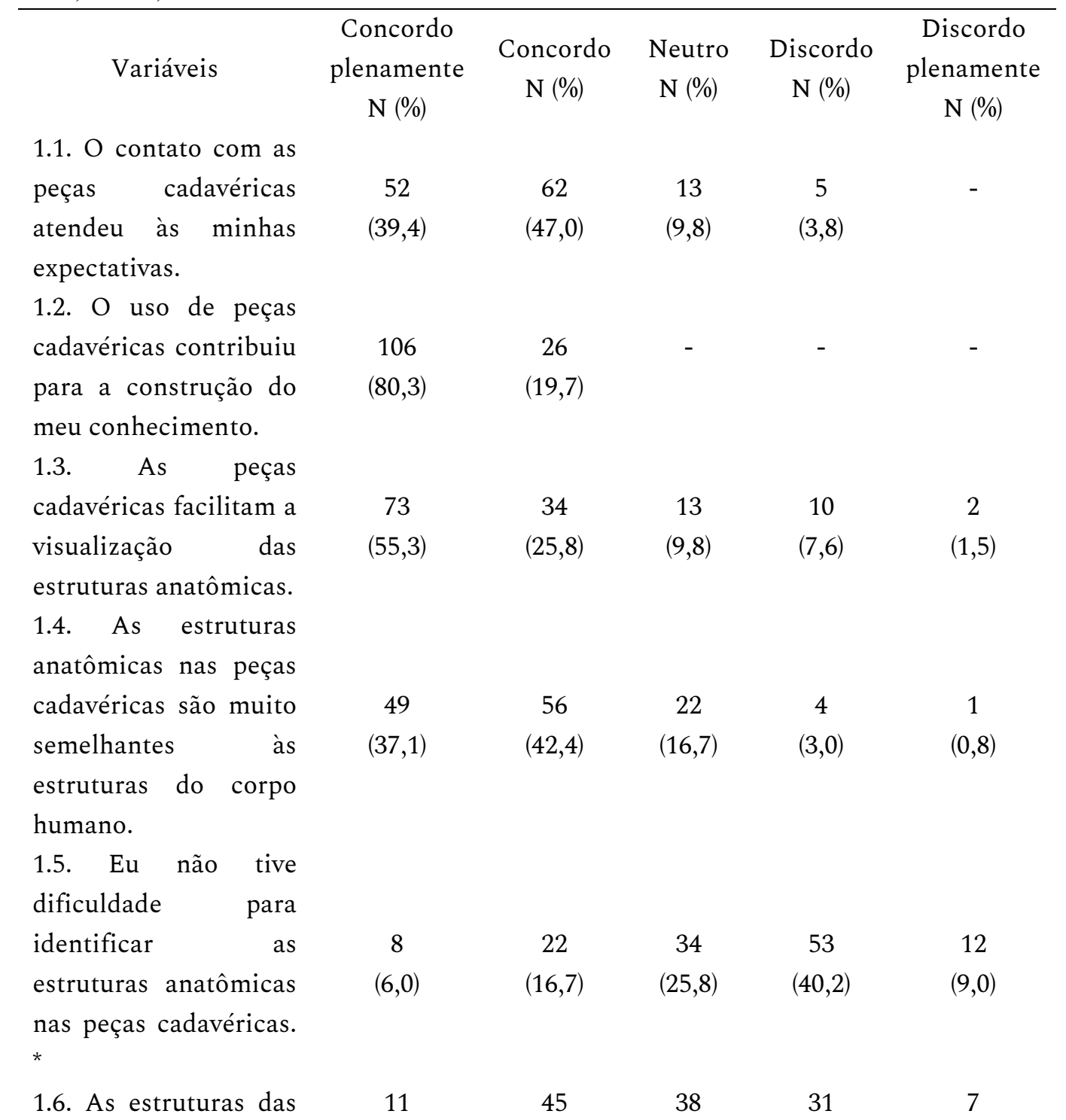




\begin{tabular}{|c|c|c|c|c|c|}
\hline peças cadavéricas & $(8,3)$ & $(34,1)$ & $(28,8)$ & $(23,5)$ & $(5,3)$ \\
\hline \multirow{4}{*}{\multicolumn{6}{|c|}{$\begin{array}{l}\text { correspondem às } \\
\text { imagens apresentadas } \\
\text { nos livros e nos atlas } \\
\text { de anatomia. }\end{array}$}} \\
\hline & & & & & \\
\hline & & & & & \\
\hline & & & & & \\
\hline \multirow{5}{*}{$\begin{array}{l}\text { 1.7. O contato com as } \\
\text { peças cadavéricas } \\
\text { despertou em mim } \\
\text { sentimentos de } \\
\text { angústia e mal-estar. }\end{array}$} & 2 & 4 & 6 & 43 & 77 \\
\hline & $(1,5)$ & $(3,1)$ & $(4,5)$ & $(32,6)$ & $(58,3)$ \\
\hline & & & & & \\
\hline & & & & & \\
\hline & & & & & \\
\hline \multirow{4}{*}{$\begin{array}{l}\text { 1.8. Eu consegui } \\
\text { manipular as peças } \\
\text { cadavéricas sem sentir } \\
\text { repulsa. }\end{array}$} & 77 & 35 & 12 & 5 & 3 \\
\hline & $(58,3)$ & $(26,5)$ & $(9,1)$ & $(3,8)$ & $(2,3)$ \\
\hline & & & & & \\
\hline & & & & & \\
\hline \multirow{2}{*}{$\begin{array}{l}\text { 1.9. Durante as aulas } \\
\text { eu não via os }\end{array}$} & 37 & 37 & 30 & 18 & 10 \\
\hline & $(28,0)$ & $(28,0)$ & $(22,7)$ & $(13,6)$ & $(7,7)$ \\
\hline \multicolumn{6}{|l|}{ cadáveres como } \\
\hline \multicolumn{6}{|l|}{ pessoas mortas e sim } \\
\hline como peças técnicas. & & & & & \\
\hline
\end{tabular}

* Não respondentes no item 1.5: n $(\%)=3(2,3)$.

$\mathrm{Na}$ Tabela 2, observam-se as respostas dos estudantes em relação às potencialidades e dificuldades do uso de peças sintéticas no ensino da anatomia em cursos de graduação em enfermagem.

Tabela 2 - Avaliação dos estudantes de enfermagem quanto ao uso de peças sintéticas no ensino da Anatomia, Ribeirão Preto/SP, Brasil, 2016-2017.

\begin{tabular}{|c|c|c|c|c|c|}
\hline Variáveis & $\begin{array}{c}\text { Concordo } \\
\text { plenamente } \\
\mathrm{N}(\%)\end{array}$ & $\begin{array}{l}\text { Concordo } \\
\mathrm{N}(\%)\end{array}$ & $\begin{array}{l}\text { Neutro } \\
\mathrm{N}(\%)\end{array}$ & $\begin{array}{l}\text { Discordo } \\
\mathrm{N}(\%)\end{array}$ & $\begin{array}{c}\text { Discordo } \\
\text { plenamente } \\
\mathrm{N}(\%)\end{array}$ \\
\hline $\begin{array}{l}\text { 2.1. O contato com as } \\
\text { peças sintéticas } \\
\text { atendeu as minhas } \\
\text { expectativas. * }\end{array}$ & $\begin{array}{c}35 \\
(26,5)\end{array}$ & $\begin{array}{c}43 \\
(32,6)\end{array}$ & $\begin{array}{c}30 \\
(22,7)\end{array}$ & $\begin{array}{c}16 \\
(12,1)\end{array}$ & $\begin{array}{c}7 \\
(5,3)\end{array}$ \\
\hline $\begin{array}{l}\text { 2.2. O uso de peças } \\
\text { sintéticas contribuiu } \\
\text { para a construção do } \\
\text { meu conhecimento. * }\end{array}$ & $\begin{array}{c}50 \\
(37,9)\end{array}$ & $\begin{array}{c}54 \\
(40,9)\end{array}$ & $\begin{array}{c}21 \\
(15,9)\end{array}$ & $\begin{array}{c}4 \\
(3,0)\end{array}$ & $\begin{array}{c}1 \\
(0,8)\end{array}$ \\
\hline $\begin{array}{l}\text { 2.3. As peças sintéticas } \\
\text { facilitam a visualização }\end{array}$ & $\begin{array}{c}46 \\
(34,8)\end{array}$ & $\begin{array}{c}55 \\
(41,7)\end{array}$ & $\begin{array}{c}19 \\
(14,4)\end{array}$ & $\begin{array}{c}11 \\
(8,3)\end{array}$ & $\begin{array}{c}1 \\
(0,8)\end{array}$ \\
\hline $\begin{array}{l}\text { das estruturas } \\
\text { anatômicas. }\end{array}$ & & & & & \\
\hline $\begin{array}{l}\text { 2.4. As estruturas } \\
\text { anatômicas nas peças }\end{array}$ & $\begin{array}{c}11 \\
(8,3)\end{array}$ & $\begin{array}{c}30 \\
(22,7)\end{array}$ & $\begin{array}{c}44 \\
(33,3)\end{array}$ & $\begin{array}{c}36 \\
(27,3)\end{array}$ & $\begin{array}{c}10 \\
(7,6)\end{array}$ \\
\hline
\end{tabular}


Uso de peças cadavéricas e modelos sintéticos no ensino da anatomia nos... $\mid 8$

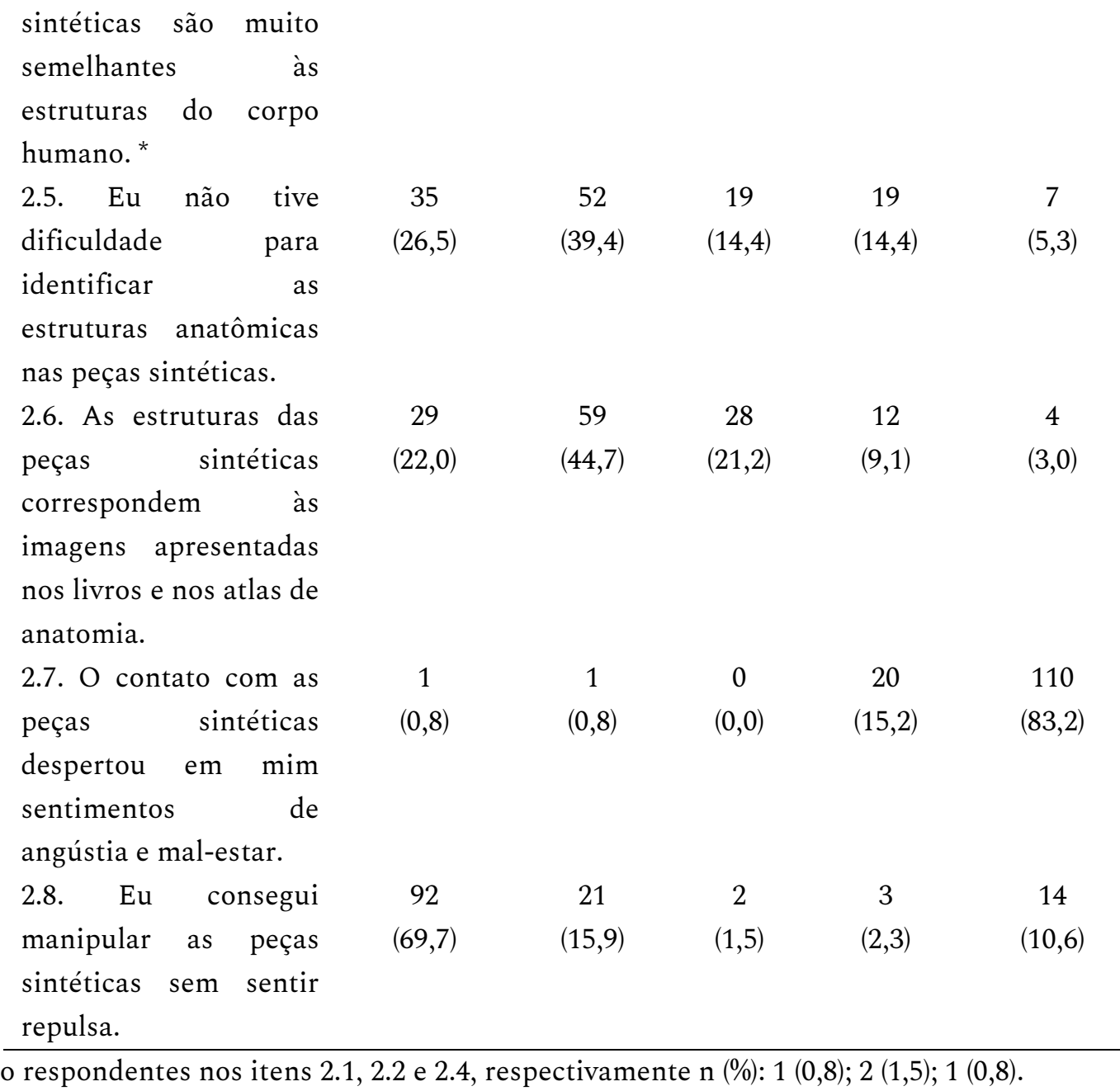

Na Tabela 3, são apresentadas as respostas dos estudantes em relação às potencialidades e dificuldades do uso concomitante de peças cadavéricas e sintéticas no ensino da anatomia em cursos de graduação em enfermagem. 
Tabela 3 - Avaliação dos estudantes de enfermagem quanto ao uso concomitante de peças cadavéricas e sintéticas no ensino da Anatomia, Ribeirão Preto/SP, Brasil, 2016-2017.

\begin{tabular}{|c|c|c|c|c|c|}
\hline Variáveis & $\begin{array}{c}\text { Concordo } \\
\text { plenamente } \\
\mathrm{N}(\%)\end{array}$ & $\begin{array}{l}\text { Concordo } \\
\mathrm{N}(\%)\end{array}$ & $\begin{array}{l}\text { Neutro } \\
\mathrm{N}(\%)\end{array}$ & $\begin{array}{l}\text { Discordo } \\
\mathrm{N}(\%)\end{array}$ & $\begin{array}{c}\text { Discordo } \\
\text { plenamente } \\
\mathrm{N}(\%)\end{array}$ \\
\hline $\begin{array}{l}\text { 3.1. Eu consegui } \\
\text { relacionar, } \\
\text { dificuldades, } \\
\text { estruturas nas peças } \\
\text { sintéticas com as das } \\
\text { peças cadavéricas. }\end{array}$ & $\begin{array}{c}21 \\
(15,9)\end{array}$ & $\begin{array}{c}47 \\
(35,6)\end{array}$ & $\begin{array}{c}23 \\
(17,4)\end{array}$ & $\begin{array}{c}34 \\
(25,8)\end{array}$ & $\begin{array}{c}7 \\
(5,3)\end{array}$ \\
\hline $\begin{array}{l}\text { 3.2. Acredito que } \\
\text { utilizar os dois tipos } \\
\text { de peças facilitou o } \\
\text { entendimento do } \\
\text { conteúdo. }\end{array}$ & $\begin{array}{c}73 \\
(55,3)\end{array}$ & $\begin{array}{c}42 \\
(31,8)\end{array}$ & $\begin{array}{c}9 \\
(6,8)\end{array}$ & $\begin{array}{c}6 \\
(4,5)\end{array}$ & $\begin{array}{c}2 \\
(1,6)\end{array}$ \\
\hline $\begin{array}{l}\text { 3.3. Acredito que o uso } \\
\text { de somente peças } \\
\text { sintéticas é suficiente } \\
\text { para a minha } \\
\text { aprendizagem. }\end{array}$ & $\begin{array}{c}1 \\
(0,8)\end{array}$ & $\begin{array}{c}2 \\
(1,5)\end{array}$ & $\begin{array}{c}1 \\
(0,8)\end{array}$ & $\begin{array}{c}34 \\
(25,7)\end{array}$ & $\begin{array}{c}94 \\
(71,2)\end{array}$ \\
\hline $\begin{array}{l}\text { 3.4. As peças sintéticas } \\
\text { são o melhor recurso } \\
\text { para o aprendizado da } \\
\text { anatomia. }\end{array}$ & $\begin{array}{c}0 \\
(0,0)\end{array}$ & $\begin{array}{c}5 \\
(3,8)\end{array}$ & $\begin{array}{c}21 \\
(15,9)\end{array}$ & $\begin{array}{c}52 \\
(39,4)\end{array}$ & $\begin{array}{c}54 \\
(40,9)\end{array}$ \\
\hline $\begin{array}{l}3.5 . \quad \text { As } \\
\text { cadavéricas são } \\
\text { melhor recurso para o } \\
\text { aprendizado } \\
\text { anatomia. }\end{array}$ & $\begin{array}{c}50 \\
(37,9)\end{array}$ & $\begin{array}{c}51 \\
(38,6)\end{array}$ & $\begin{array}{c}16 \\
(12,1)\end{array}$ & $\begin{array}{c}11 \\
(8,3)\end{array}$ & $\begin{array}{c}4 \\
(3,1)\end{array}$ \\
\hline $\begin{array}{l}\text { 3.6. Acredito que o uso } \\
\text { de somente peças } \\
\text { cadavéricas é }\end{array}$ & $\begin{array}{c}9 \\
(6,8)\end{array}$ & $\begin{array}{c}19 \\
(14,4)\end{array}$ & $\begin{array}{c}32 \\
(24,3)\end{array}$ & $\begin{array}{c}51 \\
(38,6)\end{array}$ & $\begin{array}{c}21 \\
(15,9)\end{array}$ \\
\hline $\begin{array}{l}\text { suficiente para a } \\
\text { minha aprendizagem. }\end{array}$ & & & & & \\
\hline
\end{tabular}

\section{Discussão}

As respostas dos estudantes mostram que o uso de peças cadavéricas para o ensino da anatomia, é capaz de atender às expectativas de aprendizagem, auxiliar na construção do conhecimento, sendo considerado um bom recurso para o ensino. A anatomia é uma disciplina 
Uso de peças cadavéricas e modelos sintéticos no ensino da anatomia nos... $\mid 10$

propedêutica que contribui para o desenvolvimento de competências dos estudantes de enfermagem para o cuidado em saúde, pois, permite a construção do conhecimento sobre a forma e a localização das estruturas do corpo humano correlacionando-as com suas funções ${ }^{13}$ e, coopera com o desenvolvimento de competências para as intervenções de enfermagem. Ademais, em se tratando do uso do cadáver, o estudante e o professor podem desenvolver princípios como a humanização e ética. ${ }^{14}$

Como as ciências biológicas e as ciências anatômicas passaram por uma grande fase de evolução, muitos procedimentos e tratamentos clínicos e cirúrgicos vêm acompanhando essa trajetória, o que faz com que os cuidados de enfermagem se tornem cada vez mais complexos. Para que o enfermeiro seja capaz de desempenhar seu papel, faz-se necessária a compreensão da anatomia, a qual dará a esse profissional, subsídios para executar os fundamentos que norteiam a prática como a avaliação minuciosa das condições físicas e clínicas do paciente e dos resultados dos tratamentos aplicados. ${ }^{15}$

A articulação da teoria com a prática no ensino de anatomia pode colaborar para ampliar a construção do conhecimento pelo estudante. A adoção de estratégias de ensino como a utilização de cadáveres, modelos sintéticos, e-learning, vídeos e realidade tridimensional pode favorecer a aprendizagem, uma vez que, mobiliza várias habilidades no estudante. ${ }^{6}$ Para 0 professor, a apropriação da combinação do conhecimento prático, teórico e a união de diversos métodos de ensino, pode contribuir para aulas mais dinâmicas e atrativas. ${ }^{16}$ Nesse contexto, acredita-se que o elo desses fatores com a integração entre o professor e o estudante possibilita o embasamento do futuro profissional tornando-o mais capaz ao lidar com os sistemas do corpo e, sobretudo, suas complexidades. ${ }^{16}$

A evolução e a adoção de métodos e estratégias de ensino contemporâneos no ensino em saúde, propiciaram que recursos inovadores e criativos, como as tecnologias educacionais computadorizadas, fossem utilizadas nos cursos de enfermagem. Essas tecnologias 
compreendem vídeos interativos, simulações computacionais, softwares interativos, livros-texto, atlas, imagens, modelos anatômicos sintéticos e peças orgânicas de animais não humanos ${ }^{6,8-9,17}$ capazes de contribuir com o processo de ensino-aprendizagem, facilitando com que o estudante exerça suas funções com propriedade.

No que se refere aos sentimentos dos estudantes associados ao manuseio de peças nas atividades práticas, os resultados evidenciaram que grande parte dos estudantes não sentiram angústia, mal-estar ou repulsa $(58,3 \%)$ ao entrarem em contato com peças cadavéricas, a qual pode ser considerada a primeira abordagem do estudante com o cadáver. ${ }^{18}$ Portanto, é importante ter o cuidado de vincular os aspectos técnicos aos ético-humanísticos, de forma que os estudantes desenvolvam habilidades para a competência em suas profissões sem que se esqueçam da humanização. ${ }^{14}$

A anatomia embasa a prática de enfermagem como a anamnese, o exame físico e banho no leito, a identificação de uma musculatura para aplicação de vacina, ${ }^{18}$ o desenvolvimento das responsabilidades profissionais e o compromisso ético e social, embora esses últimos, ainda sejam processos em construção no ensino-aprendizagem. ${ }^{15}$

Em relação ao uso das peças sintéticas, observou-se que essas não despertaram sentimentos de angústia e mal-estar nos estudantes, sendo essa evidência considerada uma potencialidade. Contudo, para a maioria dos estudantes, o uso apenas de peças sintéticas não é suficiente para o ensino-aprendizagem da anatomia humana.

Evidencia-se que no manuseio de estruturas sintéticas, o sentimento despertado nos estudantes, pode ser de algo irreal, artificial, falso e/ou inalterado, ${ }^{14}$ incapaz de gerar quaisquer sentimentos de repulsa ou mal-estar, ao contrário dos sentimentos despertados pelas peças cadavéricas, por meio das quais os estudantes podem apresentar sentimentos de ansiedade, expressões de nojo e medo ou até mesmo de repulsa..$^{10,12}$ 
Uso de peças cadavéricas e modelos sintéticos no ensino da anatomia nos... | 12

Quando no questionário interrogou-se se, os estudantes não viam os cadáveres como pessoas mortas e sim como peças técnicas, observou-se maior variedade de respostas, $28 \%$ dos participantes concordaram plenamente, $28 \%$, concordaram e $22,7 \%$ ficaram neutros ao responderem, denotando a importância de o professor reforçar as aprendizagens atitudinais durante o ensino de anatomia.

Nesse ponto, recomenda-se ter cautela para que os estudantes não desenvolvam uma visão excessivamente biológica e tecnicista das peças cadavéricas, com fragmentação do corpo humano, o que poderia causar uma despersonalização do indivíduo (não vivo), ${ }^{14}$ excluindo a integridade e humanização que esses corpos possuem. Na prática, essa visão biológica e tecnicista limita a assistência de enfermagem, pois, o profissional acaba dirigindo um olhar mais voltado à doença, à patologia, do que ao lado socioemocional do paciente. ${ }^{19-20}$

Nesse contexto, o professor ao planejar as atividades de ensino deve considerar a articulação entre as aprendizagens cognitivas, procedimentais e atitudinais, pois, é pertinente não compartimentar a estrutura do conhecimento, a qual na prática, nunca é aprendida e apreendida de forma separada. ${ }^{21}$

Como anteriormente descrito, a aprendizagem de atitudes deve fazer parte das ações pedagógicas do professor, posto que o ensino de princípios ou ideias éticas favorece as pessoas emitirem um juízo sobre as condutas e seu sentido. ${ }^{21}$ Outrossim, na aprendizagem de atitudes, cabe ao professor, desde o início do curso, reforçar ao estudante ingressante que a aprendizagem será construída para atingir o objetivo de formação, ou seja, o cuidado ao paciente/ser humano, o qual não é apenas um órgão ou uma patologia. Nesse sentido, a comunicação dos profissionais de saúde com os pacientes mais graves gera uma assistência voltada à técnica e aos controles de natureza fisiológica, sendo esses incapazes de lidar em sua vida profissional com sentimentos de dor e morte devido à ausência de visão integral e humanizada do paciente/família durante sua formação. ${ }^{19-20}$ 
Atualmente, as faculdades têm enfocado uma nova vertente que busca estratégias de ensino inovadoras, com vistas a inserir o estudante em uma prática pedagógica ética, crítica, reflexiva e transformadora, ultrapassando os limites do treinamento puramente técnico, para efetivamente alcançar a formação do homem como um ser histórico, inscrito na dialética da ação-reflexão-ação, propondo ao estudante a capacidade de desenvolver uma visão integral e humanizada no cuidado humano..$^{21}$

Quando os estudantes foram questionados se o contato com as peças cadavéricas atendeu as suas expectativas, $39,4 \%$ concordaram plenamente e $47 \%$ concordaram, em relação às peças sintéticas; $26,5 \%$ e $32,6 \%$ concordaram plenamente e concordaram, respectivamente.

Um estudo realizado em uma instituição de ensino no nordeste brasileiro mostrou as percepções dos estudantes em relação ao processo de ensino-aprendizagem da disciplina de anatomia; foi evidenciado que para $93,4 \%$ dos estudantes o uso de manequins sintéticos era o principal modelo adotado para as aulas práticas de anatomia. Os autores fazem um alerta com relação à falta de cadáveres nas instituições e sobre as dificuldades para sua aquisição, ressaltando a importância de outros métodos que permitam ao estudante ter mais afinidade com a anatomia e serem mais ativos no seu aprendizado. ${ }^{22}$

É esperado que os estudantes se sintam ansiosos e entusiasmados para a primeira aula de anatomia ou que possam sentir medo frente ao cadáver humano. Da mesma forma, podem não demonstrar nenhum sentimento por pensarem que será apenas mais uma disciplina contribuindo para sua formação como profissional, independentemente da expectativa dos discentes. Ansiosos, entusiasmados, assustados ou indiferentes, eles poderão apresentar dificuldades para aprendizagem da anatomia humana, entretanto, um estudo mostrou que a motivação do estudante para a aprendizagem pode melhorar o seu desempenho acadêmico. ${ }^{23}$

O uso de livros didáticos, imagens e réplicas sintéticas do corpo humano é visto, por alguns autores, como fantástico meio de estudo para os estudantes e profissionais da saúde, 
Uso de peças cadavéricas e modelos sintéticos no ensino da anatomia nos... | 14

visto que tem facilitado a busca por informações e dão uma ideia das estruturas anatômicas contribuindo assim para sua visualização..$^{3,9,18,24}$ Complementarmente, os softwares anatômicos também oferecem aos estudantes imagens tridimensionais das estruturas e são vistos como um suporte adicional para sua identificação. ${ }^{9,13,24}$

Quando perguntado se as peças sintéticas são semelhantes às estruturas do corpo humano, $33,3 \%$ dos estudantes mantiveram-se neutros na resposta e $27,3 \%$ discordaram. O interessante é que $44,7 \%$ dos participantes acreditam que as peças sintéticas correspondem às imagens apresentadas nos livros e atlas de anatomia, possivelmente pelo fato dessa representação estar mais relacionada com as questões didáticas da disciplina e que facilitam a visualização e o entendimento, do que com a representação exata das estruturas do corpo humano.

Em relação às peças cadavéricas, $42,4 \%$ dos participantes concordaram que são semelhantes às estruturas do corpo humano; $37,9 \%$ e 38,6\% concordaram plenamente e concordaram, respectivamente, que as peças cadavéricas são o melhor recurso para aprender anatomia. No entanto, no presente estudo, $40,2 \%$ dos participantes referiram ter dificuldades em identificar as estruturas anatômicas. Esse resultado remete a pensar que mesmo sendo um corpo humano, as estruturas podem sofrer alterações decorrentes do manuseio, conservação e degradação. ${ }^{13}$

Entre os estudantes, $55,3 \%$ e $31,8 \%$ dos estudantes concordaram plenamente e concordaram, respectivamente, que utilizar os dois tipos de peças (sintéticas e cadavéricas), facilitou a compreensão do conteúdo; e 35,6\% concordaram que conseguiam relacionar, sem dificuldades, as estruturas nas peças sintéticas com as das peças cadavéricas. Uma pesquisa que avaliou a percepção dos estudantes sobre o ensino da anatomia mostrou que os alunos consideram ser difícil a memorização dos nomes das estruturas, contudo, as evidências reafirmam que o uso de cadáveres facilita o aprendizado, ${ }^{18}$ resultado similar ao desse estudo.

Quando perguntados se as peças sintéticas são o melhor recurso para a aprendizagem, $40,9 \%$ e $39,4 \%$ discordaram plenamente e discordaram, respectivamente. Quando perguntados se 
somente o uso das peças cadavéricas é suficiente para a sua aprendizagem, $38,6 \%$ dos estudantes discordaram. Esses resultados reafirmam que os novos recursos de ensino podem ser usados em concomitância com as peças cadavéricas, mas não em substituição a essas pois, servem como apoio e complemento ao estudante no processo ensino-aprendizagem. ${ }^{13-15,24}$

\section{Conclusão}

Esse estudo mostrou que o uso de cadáveres no ensino da anatomia apresenta mais potencialidades quando comparado ao o uso de peças sintéticas. Embora os cadáveres venham a sofrer alterações em suas estruturas ao longo do tempo, devido ao manuseio, conservação ou degradação, os estudantes de enfermagem julgaram positivamente o uso destes para a construção do conhecimento. Observou-se que o uso de modelos sintéticos é positivo no processo de ensinoaprendizado, desde que associado às cadavéricas como complemento ao estudante.

As evidências desse estudo se limitaram ao tamanho da amostra e a inclusão de estudantes de uma instituição de ensino. Entretanto, espera-se que se possa contribuir com o planejamento e inclusão de estratégias de ensino-aprendizado da anatomia, especificamente para os cursos de enfermagem, de maneira que atendam as expectativas dos estudantes aproximandoos do exercício da enfermagem. As potencialidades e dificuldades relacionadas ao uso de peças cadavéricas e sintéticas podem ser exploradas por meio de outros instrumentos que contenham questões mais objetivas, incluindo outras instituições de ensino em enfermagem, em diferentes regiões do país e englobando os recursos tecnológicos, a fim de aprimorar os métodos de ensino.

\section{Referências}

1. Tortora GJ, Derrickson B. Princípios de anatomia humana. 10ª ed. Porto Alegre (RS): Artmed; 2017.

2. Guerrero Guzmán CC, Pérez Díaz KA, Ruíz Díaz MP, Díaz Sánchez V, Ariza Aguirre AC, Cantor Alfonso LC, et al. Restoration and conservation of anatomic pieces. Anat Cell Biol [Internet]. 2019 set [acesso em 2020 fev 13];52(3):255-61. Disponível em: http://doi:10.5115/acb.19.021 
Uso de peças cadavéricas e modelos sintéticos no ensino da anatomia nos... | 16

3. Johnston ANB, Hamill J, Barton MJ, Baldwin S, Percival J, Williams-Pritchard G, et al. Student learning styles in anatomy and physiology courses: meeting the needs of nursing students. Nurse Educ Pract [Internet]. 2015 nov [acesso em 2018 nov 09];15(6):415-20. Disponível em: https://doi.org/10.1016/j.nepr.2015.05.001

4. BRASIL. Ministério da Educação e do Desporto. Portaria no 1.721 , de 15 de dezembro de 1994. Estabelece o currículo mínimo do curso de graduação em Enfermagem. Diário Oficial da União, Brasília, 1994 dez 16. Seção 1, p. 19801-19082.

5. Nunes JT, França DJR, Felix RS, Fernandes MNF. Processo de ensino-aprendizagem no desempenho das atividades de monitoria: relato de experiência. Rev Enferm UFPE On Line [Internet]. 2014 [acesso 2018 Nov 09];8(Supl 3):4165-9. Disponível em: https://periodicos.ufpe.br/revistas/revistaenfermagem/article/view/10156/10675

6. Davis CR, Bates AS, Ellis H, Roberts AM. Human anatomy: let the students tell us how to teach. Anat Sci Educ [Internet]. 2013 [acesso em 2018 nov 09];7(4):262-72. Disponível em: https://doi.org/10.1002/ase.1424

7. Silva GR, Cortez POBC, Lopes ISL, Teixeira BACB, Leal NMS. Métodos de conservação de cadáveres humanos utilizados nas faculdades de medicina do Brasil. Rev Med [Internet]. 2016 dez [acesso 2018 nov 09];95(4):156-61. Disponível em: https://doi.org/10.11606/issn.1679-9836.v95i4p156-161

8. Haviz M, Novita N, Helmitha R. Designing and developing teaching material of human anatomy with thinking map: what is internal relevance and consistency? EDUSAINS [Internet]. 2018 [acesso em 2020 fev 13];10(1):38-45. Disponível em: http://journal.uinjkt.ac.id/index.php/edusains/article/view/7206

9. Saltarelli AJ, Roseth CJ, Saltarelli WA. Human cadavers vs. multimedia simulation: a study of student learning in anatomy. Anat Sci Educ [Internet]. 2014 [acesso em 2018 nov 09];7(5):331-9. Disponível em: https://doi.org/10.1002/ase.1429

10. Biswas R, Bandyopadhyay R. Attitude of first year medical students towards cadaveric dissection: a cross sectional study in a medical college of West Bengal, India. Int J Community Med Public Health [Internet]. 2019 jun [acesso em 2020 fev 13];6(6):2679-83. Disponível: http://dx.doi.org/10.18203/23946040.ijcmph20192343

11. Cummings SR, Hulley SB, Stewart AL. Elaboração de questionário e instrumento de coleta de dados. In: Hulley SB, Cummings SR, Browner WS, Grady D, Hearst NB, Newman TB. Delineando a pesquisa clínica: uma abordagem epidemiológica. $4^{\mathrm{a}}$ ed. Porto Alegre: Artmed; 2015. p. 265-81.

12. Mc Garvey A, Hickey A, Conroy R. The anatomy room: a positive learning experience for nursing students. Nurse Educ Today [Internet]. 2015 jan [acesso em 2020 fev 13];35(1):245-50. Disponível em: https://www.ncbi.nlm.nih.gov/pubmed/25169972 
13. Johnston AN, Hamill J, Barton MJ, Baldwin S, Percival J, Williams-Pritchard G, et al. Student learning styles in anatomy and physiology courses: meeting the needs of nursing students. Nurse Educ Pract [Internet]. 2015 nov [acesso em 2020 fev 13];15(6):415-20. Disponível em: https://www.ncbi.nlm.nih.gov/pubmed/26027548

14. Borba KP. O estudo de anatomia no ensino de enfermagem: reflexões sobre princípios éticos. Ciênc Cuid Saúde [Internet]. 2017 jan-mar [acesso em 2018 nov 09];16(1):01-6. Disponível em: http://dx.doi.org/10.4025/cienccuidsaude.v16i2.32021

15. Oliveira RCE, Tinôco JDS, Delgado MF, Andriola IC, Silva CMB, Lira ALBC. Estratégia educativa no ensino de anatomia humana aplicada à enfermagem. Av Enferm [Internet]. $2018 \mathrm{abr}$ [acesso em $2020 \mathrm{fev}$ 13];36(1):31-9. Disponível em: http://dx.doi.org/10.15446/av.enferm.v36n1.61034

16. Cocce ALR, Silveira LM, Goes FDN, Souza ALT, Stabile AM. O ensino da anatomia nas escolas de enfermagem: um estudo descritivo. Arq Ciênc Saúde [Internet]. 2017 [acesso em 2018 nov 09];24(4):08-13. Disponível em: https://doi.org/10.17696/2318-3691.24.4.2017.818

17. Góes FSN, Fonseca LMM, Camargo RAA, HaraNakata CYN, Gobbi JD, Stabile AM. Elaboração de um ambiente digital de aprendizagem na educação profissionalizante em enfermagem. Cienc Enferm [Internet]. 2015 abr [acesso em 2018 nov 09];21(1):81-90. Disponível em: http://dx.doi.org/10.4067/S071795532015000100008

18. Salbego C, Oliveira EMD, Silva MAR, Bugança PR. Percepções acadêmicas sobre o ensino e a aprendizagem em anatomia humana. Rev Bras Educ Med [Internet]. 2015 [acesso em 30 jan 2017];39(1): 23-31. Disponível em: http://dx.doi.org/10.1590/1981-52712015v39n1e00732014

19. Duarte AC, Almeida DV, Popim RC. A morte no cotidiano da graduação: um olhar do aluno de medicina. Interface (Botucatu) [Internet]. 2015 dez [acesso em 2018 nov 09];19(55):1207-19. Disponível em: http://dx.doi.org/10.1590/1807-57622014.1093

20. Jones DG, King MR. Maintaining the anonymity of cadavers in medical education: historic relic or educational and ethical necessity? Anat Sci Educ [Internet]. 2017 jan [acesso em 2018 nov 09];10(1):87-97. Disponível em: https://doi.org/10.1002/ase.1618

21. Cruz PO, Carvalho TB, Pinheiro LDP, Giovannini PE, Nascimento EGC, Fernandes TAAM. Perception of effectiveness of the teaching methods used in a medical course in Northeast Brazil. Rev Bras Educ Med [Internet]. 2019 [acesso em 2020 fev 13];43(2):40-7. Disponível em: http://dx.doi.org/10.1590/1981-52712015v43n2rb20180147

22. Oliveira PS, Lucena JD, Silva FRO, Leal KMB, Ferreira JN, et al. The teaching of human anatomy in Brazil: the reality of Northeast Brazil. Int J Anat Res [Internet]. 2017 [acesso em 2019 nov 09];5(4.2): 462329. Disponível em: https://pdfs.semanticscholar.org/e3ff/34e6212060fb580f10ffea9a622f82bf3ec1.pdf 
Uso de peças cadavéricas e modelos sintéticos no ensino da anatomia nos... | 18

23. Sturges D, Maurer TW, Allen D, Gatch DB, Shankar P. Academic performance in human anatomy and physiology classes: a 2-yr study of academic motivation and grade expectation. Adv Physiol Educ [Internet]. 2016 mar [acesso em 2018 nov 09];40(1):26-31. Disponível em: https://doi.org/10.1152/advan.00091.2015

24. Chen S, Pan Z, Wu Y, Gu Z, Li M, Liang Z, et al. The role of three-dimensional printed models of skull in anatomy education: a randomized controlled trail. Sci Rep [Internet]. 2017 [acesso em 2020 fev 13]; 7(1):575. Disponível em: https://doi.org/10.1038/s41598-017-00647-1

\section{Autor correspondente}

Angelita Maria Stabile

E-mail: angelita@eerp.usp.br

Endereço: Avenida Bandeirantes, 3900 - Ribeirão Preto - SP - Brasil

CEP: $14040-902$

\section{Contribuições de Autoria}

1 - Nathan Mesquita Penha

Concepção, planejamento, obtenção dos dados e redação do manuscrito.

\section{2 - Laura Menezes Silveira}

Interpretação dos dados, redação do manuscrito.

\section{3 - Fernanda dos Santos Nogueira de Góes}

Revisão crítica do manuscrito.

\section{4 - Angelita Maria Stabile}

Concepção e planejamento do projeto de pesquisa, interpretação dos dados, aprovação da versão final do manuscrito.

\section{Como citar este artigo}

Penha NM, Silveira LM, Góes FNS, Stabile AM. Uso de peças cadavéricas e modelos sintéticos no ensino da anatomia nos cursos de enfermagem. Rev. Enferm. UFSM. 2020 [Acesso em: Anos Mês Dia]; vol.10 e35: 1-18. DOI:https://doi.org/10.5902/2179769235146 